\title{
Kurzbeiträge
}

Leo Becker, Uwe Jürgenhake, Christian Paqué, Peter Schnittfeld

\section{Gruppenarbeit bei der Rasselstein Hoesch GmbH}

Das Pilotprojekt Entfettungslinien

\section{$1 \quad$ Einführung}

Mit dem folgenden Beitrag wird der Prozess der Einführung von Gruppenarbeit in einem Unternehmen der Stahlverarbeitung geschildert. Es ist Teil eines umfassenden Prozesses der kontinuierlichen Verbesserung der internen Abläufe und Strukturen. „Prozesse bewusst machen, Mitarbeiterpotentiale nutzen, Eigenverantwortung stärken" ist das Motto. Gruppenarbeit steht am Ende dieses Weges, der mit der Einführung einer Teamstruktur in der Aufbauorganisation begann. Weitere Stufen waren: „Führen mit Zielen“, Delegation von Verantwortung an die Basis, Einführung von autonomer Wartung und werksvernetzter Qualitätsteams, Einführung eines neuen Lohnsystems und Flexibilisierung der Arbeitszeit. Es ist daher nicht nur hinsichtlich der weitgehenden Definition von Gruppenarbeit ein sehr anspruchsvolles Vorhaben, sondern vor allem bei der Verzahnung verschiedener Elemente der Personal- und Organisationsentwicklung, womit ein sehr ganzheitliches Konzept verbunden ist. Insofern hebt sich die Rasselstein Hoesch $\mathrm{GmbH}$ aus der Masse derjenigen Unternehmen heraus, die meinen, mit Insellösungen oder isolierten Einzelmaßnahmen könne man die Menschen im Betrieb bewegen oder die gar zu Gunsten einer strafferen Führung die Freiheitsgrade der Ausführenden vor Ort an den Anlagen wieder zurücknehmen.

\section{Vorgeschichte und Historie}

Die Rasselstein Hoesch $\mathrm{GmbH}$ ist eine Tochter der Thyssen Krupp Stahl AG. An den beiden Standorten in Andernach und Dortmund sind insgesamt knapp 2.500 Mitarbeiter beschäftigt.
Es werden knapp 1 Million Tonnen Weißblech pro Jahr gefertigt. Die beiden Entfettungslinien, um die es hier geht, sind inmitten des Produktionsflusses am Standort Andernach angesiedelt. Kaltgewalztes Band wird hier gereinigt, damit es in der nächsten Verarbeitungsstufe geglüht werden kann. Obwohl fest im Stofffluss des Unternehmens vertaktet, sind die Entfettungslinien doch ein räumlich und sachlich eigenständiges $\mathrm{Ar}$ beitssystem. Kleine Zwischenlager stellen sicher, dass auch zeitlich eine gewisse Flexibilität gegeben ist. 55 Beschäftigte arbeiten in diesem Bereich. Neben den fünf Schichtbelegschaften gibt es noch je einen Schichtkoordinator und einen Tageskoordinator. Diese letzteren sind auch für die Haubenglühen zuständig. Gemeinsam mit dem Glühbereich (2 Hauben-, 2 Durchlaufglühen) bilden die Entfettungslinien das „Team EntfettenGlühen".

Die Überlegungen, Gruppenarbeit einzuführen, konkretisierten sich im Herbst 1996. Das Vorhaben war eingebettet in die Unternehmensund Führungskultur des Unternehmens die durch Zusammenarbeit, Eigenverantwortlichkeit, flache Hierarchien, Integration von Aufgaben und permanente Weiterbildung geprägt ist. Defizite gab es vor allem noch in den gewerblichen Bereichen der Produktion. Insofern lag Gruppenarbeit als konsequente Fortsetzung des Teamgedankens nahe und wurde auch vom Vorstand ausdrücklich gewünscht.

Der konkrete Anlass im Team „EntfettenGlühen“ zur Veränderung der Arbeitsorganisation lag vor allem in einer nicht optimalen Zusammenarbeit und Arbeitseinstellung an den Anlagen. Ein wichtiger Aspekt war auch die Integration der Mannschaften der beiden Entfettungslinien. Historisch gewachsen war eine Unterteilung der Schichtmannschaften nach den beiden Entfettungslinien, obwohl diese direkt nebeneinander stehen. Stellenweise war daraus gar eine unfruchtbare Rivalität entstand. Mit der Gruppenarbeit sollten auch diese beiden Mannschaften zu einer 
Schichtmannschaft verschmolzen werden.

Die Entfettungslinien waren zum damaligen Zeitpunkt nicht gerade das Aushängeschild im Unternehmen. Die Mannschaft galt als schwierig, die innerbetriebliche Anerkennung eher gering. Mit Gruppenarbeit hier zu beginnen, schien ein Risiko zu sein. Der Teamleiter argumentierte jedoch anders:

„An dieser Anlage lässt sich viel bewegen, die Potentiale sind da, und wenn wir es hier schaffen, mit dieser Mannschaft, dann schaffen wir es auch im gesamten Werk“.

Es wurde zunächst, im Herbst 1996, ein Arbeitskreis gebildet. In diesem waren alle wichtigen Gruppen vertreten: die Teamleitung, der Tages- und die Schichtkoordinatoren, die Belegschaft, die Personalabteilung, der Betriebsrat. Zunächst einmal wurden von diesem Arbeitskreis aus wichtige Grundlagen für die Einführung von Gruppenarbeit geschaffen. Dazu gehörte die Umstellung des Schichtsystems von 4,5- auf 5schichtige Fahrweise ${ }^{1}$ sowie die Erstellung einer Qualifizierungsmatrix zur Anlernung auf weiteren Arbeitsplätzen. Trotzdem trat die Entwicklung der Gruppenarbeit im Frühjahr 1998 auf der Stelle. Es fehlte die klare Definition der zu klärenden Fragen sowie ein Konzept, welches die notwendigen Arbeitsschritte in einem sinnvollen Arbeitsprogramm zusammenfasste. Mit der Soziale Innovation research \& consult $\mathrm{GmbH}$, einer Beratungsgesellschaft die aus der Sozialforschungsstelle Dortmund hervorging, wurde gemeinsam bis zum 18.06.99 ein Konzept zum weiteren Vorgehen erarbeitet.

\section{Definition und Ziele der Gruppenarbeit}

Zunächst einmal wurde auf mehreren Kick-OffVeranstaltungen der Belegschaft und den Führungskräften das Anliegen der Gruppenarbeit verdeutlicht. Dabei wurden im Wesentlichen die bisherigen Arbeitsergebnisse, das weitere Vorgehen und vor allem der hohe Gestaltungsbedarf der Gruppenarbeit durch die Mannschaften selbst verdeutlicht.

\section{Warum Gruppenarbeit?}

- besseres Einbringen des Potentials des einzelnen Mitarbeiters

- positives soziales Verhalten

- Miteinander - nicht gegeneinander

- Stärkere Identifikation mitden Unternehmenszielen

- Steigerung des Selbstwertgefühls

- Erhöhung der Eigenverantwortlichkeit

- Gruppenarbeit = eigentliche Teamarbeit

- Langfristige Sicherung der Arbeitsplätze

- Interessantere Arbeitsaufgaben

- Steigerung der Produktivität

- Erhöhung der Motivation

- Gemeinsame Zielverfolgung aller Mitarbeiter

- konsequente Fortführung des Teamgedankens

- Verbesserte Form der Arbeitsorganisation, um in Zukunft die gesteckten Unternehmensziele zu erreichen.

Von Beginn an war es kein Ziel, über eine neue Arbeitsorganisation Personal an den Anlagen einzusparen. Die natürlich erhofften wirtschaftlichen Effekte sollten sich ausschließlich über eine reibungsärmere, besser abgestimmte, verantwortungsbewusstere und qualifiziertere Arbeitsweise einstellen. Dies hat wesentlich dazu beigetragen, dass sich die Mannschaft auf den Prozess eingelassen hat.

\section{Ziele von Gruppenarbeit}

Unternehmensziele:

- Kostenreduzierung

- Vermeidung von Verschwendungen

- Sicherung des Gewinns

- Steigerung der Produktivität

- Langfristige Sicherung der Wettbewerbsfähigkeit

- Verbesserung der Qualität

- Null Unfälle

- niedrige Fehlzeitenquote

- Verbesserung des Informationsaustausches

- Verbesserung der Mitarbeiterqualifikationen

- Mehr Flexibilität beim Personaleinsatz

- Mehr Verbesserungsvorschläge

Mitarbeiterziele:

1 Mit der 5-schichtigen Fahrweise beträgt die durchschnittliche wöchentliche Arbeitszeit 31,5 Stunden, 3,5 Stunden weniger als die tarifliche Arbeitszeit. Zum 1.2.1998 wurden den Mitarbeitern daher angeboten, ihre arbeitsvertragliche Arbeitszeit entsprechend zu reduzieren. 42 Prozent der Belegschaft an den Entfettungslinien haben hiervon Gebrauch gemacht. Damit waren geringe Lohneinbußen verbunden. 


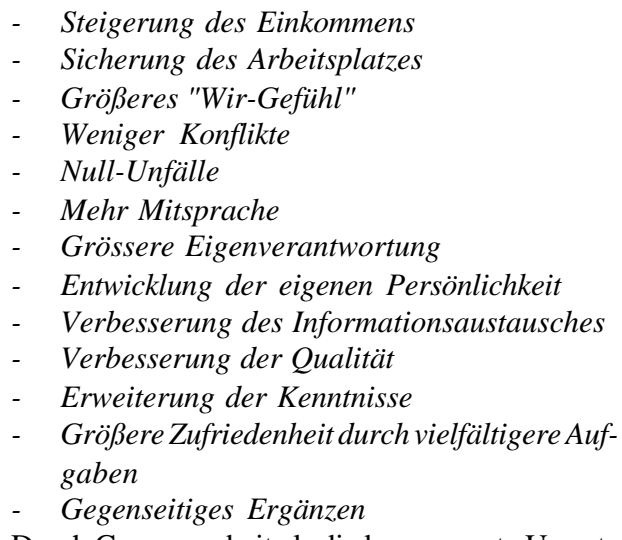

Durch Gruppenarbeit als die konsequente Umsetzung der Teamorganisation sollen die Fähigkeiten und Kenntnisse der Mitarbeiter stärker genutzt werden. Sie definiert sich als Arbeit in einer selbständigen, mit vielseitigen Funktionen ausgestatteten Arbeitseinheit, die in sich abgeschlossen sein muss. Es kam daher ein großes Spektrum möglicher Gruppenaufgaben in Betracht, die es zu konkretisieren galt. Neben ausführenden Tätigkeiten sind dabei auch personalbezogene und dispositive Tätigkeiten als mögliche Gruppenaufgaben von Beginn an mit in Betracht gezogen worden. Als Endziel war geplant, dass alle Aufgaben von den Gruppen erledigt werden sollen. Die Entscheidung darüber wurde in den neu zu gründenden Arbeitskreis (siehe unten) verlagert, dem damit eine hohe Entscheidungskompetenz zukam.

Das Spektrum möglicher Gruppenaufgaben umfasst folgende Punkte:

- Qualität

- Produktion, Wartung/Reparaturen

- Ausbringen, Kostenreduzierung

- Beschaffung von Betriebs- und Hilfsstoffen

- Arbeitssicherheit

- Anlagenbezogene Disposition, Materialfluss steuern

- Personaleinsatz, Urlaubsplanung

- Arbeitsabläufe optimieren

- Umsetzung von Verbesserungsvorschlägen

- Einarbeitung neuer Mitarbeiter, Schulung und Weiterbildung

- Informationsaustausch

- Ordnung und Sauberkeit Betreuung der Auszubildenden

- Gruppensprechertätigkeit, Moderation von Gruppensitzungen
- Kommunikation mit Mitarbeitern an Vor- und Nachanlagen

- Zwischenmenschliche Konfliktlösung

- Soziale Verantwortung tragen

\section{Vorgehensweise}

Ausgehend von diesem Rahmen wurde die weitere Entwicklung der Gruppenarbeit an den Entfettungslinien weiter vorangetrieben.

\subsection{Die Prozessbegleiter (Aufgaben, Anforderungen, Qualifizierung)}

Zur ständigen Begleitung der Aktivitäten wurden drei Prozessbegleiter ernannt. Diese haben die Aufgabe, den Prozess der Entwicklung der Gruppenarbeit, aber auch die Phase der Einführung zu begleiten sowie die Gruppenarbeit nach erfolgreicher Etablierung weiter zu entwickeln, permanent zu ,,pflegen“.

Die Aufgaben der Prozessbegleiter sind damit vielfältig. Sie beginnen mit der Koordinierung und Moderierung der Arbeitskreissitzungen. Die Ergebnisse und Maßnahmen dieser Sitzungen müssen von ihm entweder selbst umgesetzt werden, oder aber die Umsetzung muss von ihm organisiert werden. Ähnliches gilt für die anstehenden Qualifizierungsmaßnahmen. Vor allem aber müssen sie durch gute inhaltliche Vorbereitung und durch geschickte Moderation der Sitzungen dafür sorgen, dass die Ausgestaltung der neuen Arbeitsorganisation Formen annimmt und dabei die Interessen aller Beteiligten sorgsam austariert werden.

Umfangreiche inhaltliche und soziale Aufgaben haben sie auch in der Startphase der Gruppenarbeit, wenn es gilt, die Gruppensprecher und Führungskräfte zu unterstützen, bei Konflikten zu helfen, den Erfahrungsaustausch zwischen den Gruppen in Gang zu setzen oder die Teamleitung über die Entwicklung zu informieren.

Aus diesem Aufgabenspektrum ergeben sich die Qualifikationsanforderungen an die Prozessbegleiter. Sie müssen zunächst einmal die betrieblichen Zusammenhänge kennen, zugleich aber einen gewissen Abstand zu den betrieblichen Akteuren haben, d.h. möglichst keine Vorgesetztenfunktion ausüben. Darüber hinaus sind die wichtigsten Grundprinzipien der angestrebten neuen Arbeitsorganisation zu kennen und die Gründe für den betrieblichen Veränderungsprozess zu beherrschen. Sie müssen auch den Wandel der 
Rolle der betrieblichen Führungskräfte thematisieren und vermitteln können. Neben diesen fachlich-inhaltlichen Anforderungen ist eine umfangreiche Methoden- und Sozialkompetenz notwendig.

Die Prozessbegleiter im Team „Entfetten-Glühen" repräsentieren sowohl das Personalwesen als auch die Technik. Sie sind Mitglieder des Teams, jedoch in keiner hierarchischen Funktion. Diese Benennung spezieller Ansprechpartner und Koordinatoren des Einführungsprozesses hat sich überaus bewährt. In der Übertragung der neuen Arbeitsorganisation auch in andere Bereiche des Unternehmens wird aus dem Duo ein Trio: ein Betriebsrat stößt noch hinzu. Die betriebliche Interessenvertretung ist damit direkt eingebunden und umfassend informiert. Etwaige Probleme können dezentral und zeitnah gelöst werden.

\subsection{Der Arbeitskreis Gruppenarbeit und seine Tätigkeit}

Der bestehende „Arbeitskreis Gruppenarbeit" wurde nach den Kick-Off-Veranstaltungen neu zusammengesetzt. Jeweils ein Vertreter der 5 Schichtmannschaften, zwei Vertreter der Schichtkoordinatoren, der Tageskoordinator sowie der Betriebsrat bildeten den neuen Arbeitskreis. Der Teamleiter war nicht mehr vertreten. Damit wurde deutlich gemacht, dass die Konkretisierung der Gruppenarbeit ausschließlich in den Händen derjenigen lag, die sie auch später mit Leben zu erfüllen hatten.

Moderiert und koordiniert wurden die Sitzungen des Arbeitskreises von den Prozessbegleitern. Auf Wunsch der Schichtkoordinatoren wurde der Arbeitskreis aufgestockt: Statt zwei arbeiteten alle fünf Schichtkoordinatoren mit. Die Vertreter der Schichtmannschaften waren zunächst diejenigen, welche auch im ersten Arbeitskreis teilgenommen hatten. Nachdem die Gruppen ihre Gruppensprecher gewählt hatten (siehe unten), änderte sich entsprechend die Zusammensetzung des Arbeitskreises. Die jeweiligen Schichtvertreter hatten gemeinsam mit den Prozessbegleitern die Aufgabe, die anderen Kollegen der Schicht über die Entwicklungen im Arbeitskreis zu informieren und deren Meinung einzuholen. $\mathrm{Zu}$ diesem Zweck wurden in regelmäßigen Abständen Schichtversammlungen organisiert, an denen die Prozessbegleiter über die jeweils letzte Arbeitskreissitzung informierten.
Der Arbeitskreis beschäftigte sich mit allen wichtigen Fragen der Ausgestaltung der neuen Arbeitsorganisation. Es lassen sich dabei zwei große Themenblöcke identifizieren:

\section{Aufgabenverteilung}

Hierbei ging es darum, zu klären, welche Aufgaben die Gruppen und welche die Schichtkoordinatoren in Zukunft übernehmen sollen. Dabei ging es auch um die Verlagerung von Tätigkeiten in die Gruppen, die traditionell in den Verantwortungsbereich der Schichtkoordinatoren fielen. Damit war direkt auch die Rolle und Funktion der Schichtkoordinatoren im Arbeitssystem angesprochen. Mittels einer Aufgabenmatrix wurde Punkt für Punkt festgelegt, welche konkreten Aufgaben in Zukunft die Gruppe zu übernehmen hat und welche die Schichtkoordinatoren haben. Auch die Funktion des Gruppensprechers wurde auf diese Art und Weise beschrieben. Diese Aufgabenmatrix war nicht endgültig, ggf. konnten weitere Aufgaben auf die Gruppe übertragen werden.

2. Qualifikationsbedarf und Qualifizierung Ausgehend von der Definition der Gruppenaufgaben wurden die hierfür notwendigen Qualifikationen diskutiert und die notwendigen Schulungen geplant. Dabei wurde rasch deutlich, dass das traditionelle Anlernen am Arbeitsplatz nicht mehr ausreicht, um die für die Gruppenarbeit notwendigen Qualifikationen zu erwerben. Das individuelle Anlernen für die verschiedenen Arbeitsplätze hat noch seine Funktion in der Erhöhung der Einsatzflexibilität an den Anlagen, in dem Umfang wie die Einzelnen es wollen und können. Hierfür wurden individuelle Anlernpläne erstellt.

Hinzu kommen jedoch weitere Qualifikationen, welche für das Funktionieren der Gruppe wichtig sind, wie Methoden- und Sozialkompetenz, aber auch fachliche Qualifikationen, die für den Arbeitsvollzug wichtig sind. Hierzu gehört anlagentechnisches Wissen, das Wissen über die Rolle der Anlage im gesamten Produktionsfluss des Werkes oder die Schnittstellen zu den direkten vor- bzw. nachgelagerten Anlagen. Hierzu wurden sowohl theoretische Schulungen durchgeführt als auch Betriebsbegehungen oder gezielte Kommunikation mit den Kollegen der Nachbaranla- 
gen organisiert. Durch diese Maßnahmen wurde das Arbeitsprozesswissen der Gruppen systematisch erhöht. Das vom Arbeitskreis entwickelte Qualifizierungsprogramm wurde von der Belegschaft akzeptiert. Der generelle Wunsch nach Weiterbildung ist sogar stark ausgeprägt.

Zur Perspektive des Arbeitskreises: Nach Klärung der wichtigen Punkte und Lösung der in der Anlaufphase der Gruppenarbeit entstandenen Probleme zeichnet es sich ab, dass der Arbeitskreis an Bedeutung verliert. Bereits nach einem halben Jahr wurden die Treffen seltener und es ist davon auszugehen, dass er von den regelmäßigen Treffen der Gruppensprecher abgelöst werden wird.

\subsection{Der Gruppensprecher}

Der Gruppensprecher jeder Schicht wurde im Rahmen des Grundlagenseminars von der Schicht in geheimer Wahl für die Dauer eines Jahres gewählt. Die Aufgaben des Gruppensprechers wurden vom Arbeitskreis im Rahmen der Erstellung der Aufgabenmatrix wie folgt definiert.

Aufgaben des Gruppensprechers

- Interessen der Gruppen vertreten

- Bei Konflikten schlichtend einwirken

- Vorbereitung und Durchführung von Gruppengesprächen, Dokumentation der Ergebnisse

- Verantwortlich für Informationsfluss

- Alle Mitarbeiter in Gespräche mit einbeziehen

- Entscheidungen anstreben, die von allen Gruppenmitgliedern getragen werden

- Probleme von sich aus ansprechen

- Ansprechpartner für alle Gruppenmitglieder

- Kontakt zum Schlichtkoordinator

Deutlich wird, dass der Gruppensprecher zwar eine Fülle von Aufgaben hat, er aber keine hierarchische Funktion übernimmt. Dies wird nicht nur durch die jährliche (Wieder)Wahl sichergestellt, sondern auch dadurch, dass er explizit keine Personalverantwortung und auch keine Produktionsentscheidungen trifft. Er organisiert und koordiniert die Gruppe, inklusive der damit verbundenen sozialen Aufgaben.

\subsection{Das Grundlagenseminar}

Das Grundlagenseminar bedeutete für die fünf Gruppen (Schichten) der Entfettungslinien den eigentlichen Auftakt der Gruppenarbeit. Nachdem sie zuvor in eher kurzen Treffs die Arbeit des Arbeitskreises mit verfolgen konnten, sich in ihrer konkreten Arbeit aber noch kaum etwas spürbar geändert hatte, waren sie nun als Gruppe gefordert. In einer ganztägigen Veranstaltung, die von den Prozessbegleitern in Zusammenarbeit mit der Abteilung Weiterbildung vorbereitet und moderiert wurde, ging es darum, sich als Gruppe zu finden und zu definieren (Aufstellen von Gruppenregeln), Techniken zu erlernen, um Konflikte konstruktiv anzugehen sowie den Gruppensprecher zu wählen.

Durch dieses Seminar hatte die Gruppe die Möglichkeit, erstmals auch gruppenbezogene Akzente zu setzen. Dies äußerte sich in der Aufstellung von verbindlichen gruppeneigenen Regeln. Obwohl Schnittmengen in den Regeln zwischen den Gruppen auftreten, sind doch auch deutliche Akzentuierungen zu verzeichnen.

\section{Beispiele einer Gruppenregel (Schicht 5)}

- Wir wollen einander zuhören und den Anderen ausreden lassen.

- Wir wollen fair zueinander sein (z.B. jedem Hilfestellung anbieten, Hilfsbereitschaft nicht ausnutzen).

- Wir wollen zuverlässig und pünktlich sein.

- Wir wollen uns gegenseitig informieren.

- Wir wollen unseren Arbeitsplatz sauber und ordentlich hinterlassen.

- Wir wollen Konflikte ansprechen und sachlich lösen.

- Wir wollen qualitätsbewusst arbeiten.

- Wir wollen uns an Arbeitssicherheitsvorschriften halten.

- Wir wollen unsere Ziele im Auge behalten und bei Bedarf aktualisieren

- Wir wollen die Regeln bei Bedarf überprüfen und evtl. ergänzen.

Die Gruppenregeln wurden, versehen mit den Unterschriften aller Gruppenmitglieder, an eigens aufgehängten Infotafeln zum Aushang gebracht. Diese Infotafeln dienen auch der Information über die Arbeit der Gruppen und damit dem Austausch zwischen den Gruppen.

\subsection{Der, ,neue“ Schichtkoordinator}

Mit der Einführung der Gruppenarbeit änderte sich auch die Rolle des Schichtkoordinators. Durch die aktive Einbeziehung der Schichtkoordinato- 
ren an der Gestaltung konnten die vorhandenen Befürchtungen ausgeräumt, die Chancen entdeckt und die Schichtkoordinatoren zu Förderer der neuen Arbeitsorganisation gemacht werden.

Durch die Entwicklung der Aufgabenmatrix wurden die Veränderungen in der Arbeitsteilung zwischen Mannschaft und Schichtkoordinatoren im Prinzip bereits festgelegt. In einer separaten Sitzung formulierten die Schichtkoordinatoren auf dieser Grundlage ihre zukünftigen Fach- und Führungsaufgaben.

\subsection{Entlohnung}

Damit Gruppenarbeit erfolgreich sein kann, bedarf es eines entsprechenden Lohnsystems. Mit der Einführung der Gruppenarbeit an den Entfettungsanlagen wurde auch ein neues Lohnsystem eingeführt. Dieses trat zum 1.4.1999 an den Entfettungslinien in Kraft, drei Monate nachdem die Gruppenarbeit offiziell gestartet worden war. Die Ziele diese Lohnsystem lauten wie folgt:

- Anforderungsgerechte Grundeinstufung in Arbeitsbereiche

- Leistungsgerechte Teamprämie

- Nutzung von Kenntnissen und Erfahrungen der Mitarbeiter

- Förderung von Qualifikation und Flexibilität

- Verbesserung des Informationsaustausches und der Zusammenarbeit

- Verbesserung der Produktionsqualität und der Anlagenzustände

- Anreize für den Kontinuierlichen Verbesserungsprozess schaffen.

Um diese Ziele zu erreichen, wurde ein Lohnsystem mit drei Komponenten konzipiert. Diese sind im Einzelnen:

- Grundentgelt

- Teamprämie

- Persönliche Zulagen.

Das Grundentgelt entspricht einer Lohngruppe des Tarifvertrages. Es erfolgt eine summarische Einstufung durch eine paritätisch besetzte Kommission. Die Prämie enthält bestimmte Prämienbezugsgrößen wie Zeitnutzgrad, Durchsatz je Betriebsstunde oder Grad der Nacharbeit. Mit diesen Bezugsgrößen wird die individuelle Leistung jeder Schicht gemessen. Das wichtige an dieser Teamprämie ist, dass sie schichtübergreifend berechnet wird. Alle Schichten erhalten somit dieselbe Prämie. Dadurch ist jede Schicht bemüht eine optimale Schichtübergabe zu gewährleisten und die schichtübergreifende Zusam- menarbeit wird gefördert. Die persönliche Zulage berechnet sich aus Vielseitigkeit, Arbeitssorgfalt und Initiative/Zusammenarbeit. Mit dieser Zulage wird es erstmals möglich die individuelle Leistung eines Mitarbeiters zu messen. Beurteilungsmerkmale wie Zusammenarbeit/Kooperation und Informations-/Erfahrungsaustausch lassen auch eine Bewertung zu, wie sich ein Mitarbeiter in die Gruppe einbringt. Das Lohnsystem ist damit eine Rahmenbedingung, die zum Gelingen einer erfolgreichen Gruppenarbeit beiträgt.

\section{$4 \quad$ Erste Erfahrungen}

Gut ein halbes Jahr nach dem offiziellen Start der Gruppenarbeit waren die ersten Erfahrungen durchweg sehr positiv. Stimmen, die wieder zur alten Arbeitsorganisation zurück wollen, gab es keine. Dies gilt sowohl für die Teamleitung selbst als auch für die direkt Betroffenen, die Schichtkoordinatoren und die Belegschaften. Was ist es nun, dass die bisher recht einhellige Bewertung ausmacht? Worin werden die Stärken der neuen Organisationsform gesehen? Und welche Probleme traten bisher auf?

Der Stellenwert des Bereiches Entfetten im Werk war früher gering. Wer dorthin versetzt worden ist, hat schnell wieder weg gewollt. Dieser Trend hat sich umgekehrt. Die Belegschaft will bleiben, und es gibt bereits neugierige Anfragen aus anderen Teilen des Werkes, was denn dort los sei.

In den Gruppen wird ein Wandel der Arbeitseinstellung konstatiert. Die Gruppen kümmerten sich um Dinge, über die früher wohl gemeckert worden wäre, die man aber nicht zu ändern versucht habe. Die Identifikation mit der Anlage und der Arbeit ist nun gestiegen, entsprechend auch die Bemühungen, Missstände abzubauen. Eine Gruppe ist zum Beispiel so weit gegangen, von sich aus Kontakt zur Tandemstraße (der direkt vorgelagerten Anlage) herzustellen und über Probleme der Weiterverarbeitung bestimmter Ringe zu sprechen und Lösungen und Umgangsweisen (Information,...) zu finden. Auch die zwischenmenschlichen Beziehungen sind wesentlich besser geworden. Zwischen einzelnen Schichten gibt es allerdings nach wie vor Konflikte, wenngleich weniger als vorher und auf einer sachlicheren Ebene. Gruppengespräche finden in der Regel alle 4 Wochen für jeweils 2 Stunden statt. Der Termin wird in der Gruppe abgestimmt. Die 
Schichtkoordinatoren nehmen an den Treffen teil, und vertreten bei Abwesenheit auch den Gruppensprecher. Betriebliche Experten nehmen auf Einladung der Gruppen teil. An der Entfettungsanlage 1 gab es z.B. seit geraumer Zeit große Probleme mit dem Bandverlauf. Was den Ingenieursspezialisten zuvor nicht gelang, konnte gemeinsam in der Gruppensitzung gelöst werden. Externe Reparaturkosten in Höhe von 25.000,$D M$ wurden so gespart.

Die Themen der Gruppen in der Anfangsphase

- Ordnung und Sauberkeit - Reinigungsbereiche der einzelnen Anlagenplätze und der gesamten Schicht

- Rotationsplan - Wer wechselt wann an welchen Arbeitsplatz

- Überarbeitung des Sicherheitshandbuches

- Waschmöglichkeiten an der Entfettung

- Überarbeitung des Expertenhandbuches

- Optimierung des Quertransportes

- Zielvorgabe für den Monat April "Sollerfüllung der Produktion und Qualitätsvorgaben" "Sicheres Arbeiten! Ziel: Null Unfälle"

- Kosteneinsparung (z.B. Hilfs- und Betriebsstoffe, Werkzeuge)

- Verbesserung der Produktionsleistung

- Optimierung des Abpressrollenwechsels an den Entfettungen

- Materialtransport von Tandemstraße 2 zur Entfettung

- Aufbewahrung von Fundsachen (z.B. Werkzeug)

Die Gruppen selbst betonen ihre größere Entscheidungsfreiheit: es würden mehr Entscheidungen - auch ohne Rücksprache - von ihnen selbst getroffen. Die Gruppengespräche werden begrüßt und die insgesamt höhere Qualifikation habe die Arbeit erleichtert: man wisse mehr über die Anlage und sei sicherer in den Entscheidungen. Dies erleichtere die Verbesserung der Anlagenleistung.

Das Rotationssystem entwickeln die Gruppen selbst, ebenso wie das Anlernen auf neue Arbeitsplätze. Allerdings bringe die Rotation- so eine Gruppe - zunächst einmal auch Nachteile mit sich: am alten Stammarbeitsplatz habe man sich sicherer gefühlt. Diese Sicherheit könne sich aber mit der Zeit auch an den neuen Arbeitsplätzen einstellen. Anfangsprobleme in der Rotation werden zudem auf den noch unzureichenden Stand der Anlernung zurückgeführt. Eine andere Grup- pe sieht die gleichen Anfangsprobleme, bewertet den Arbeitsplatzwechsel jedoch bereits nach einem halben Jahr als sehr positiv. Dabei sei der Arbeitsplatzwechsel nur einen kleiner Teil der Gruppenarbeit.

Die Schichtkoordinatoren haben sich als wichtiger Motor zur Entfaltung der Gruppenarbeit herausgestellt (,Der Schlüssel sind die Schichtkoordinatoren“"). Je stärker sie sich bemühen, die Gruppen bei der Übernahme von neuen Aufgaben zu unterstützen, desto besser entwickelt sich die neue Aufgabenteilung. In die Vorbereitung und Begleitung der Schichtkoordinatoren muss - so die Erfahrung - fast ebenso viel investiert werden wie in die Gruppen selbst.

Die Prozessbegleiter: Die Prozessbegleiter haben sich als wichtige Stütze der neuen Arbeitsorganisation erwiesen. Sie lenken den Prozess in geordnete Bahnen, verhindern das Einschlafen, sind die Motoren des Prozesses. Ebenso wie die Schichtkoordinatoren beim eigentlichen Start von großer Bedeutung sind, sind es die Prozessbegleiter in der Phase der Entwicklung und Startvorbereitung, wenngleich sie auch danach eine wichtige Rolle spielen. An ungeeigneten Prozessbegleitern kann auch der Transfer scheitern.

Zur ökonomischen Rentabilität: Deutliche Verbesserungen von betriebswirtschaftlichen Kennziffern konnten binnen eines halben Jahres noch nicht realisiert werden. Aber auch keine Verschlechterung, sagen die Verantwortlichen im Team und verweisen darauf, dass angesichts der doch erheblichen Veränderungen durch die neue Arbeitsorganisation eigentlich mit Einbrüchen in der Leistung in dieser Anfangsphase zu rechnen gewesen wäre. Sie rechnen damit, dass sich zählbare Erfolge erst nach und nach einstellen werden. Es ist im Unternehmen aber akzeptiert, dass Veränderung einer Unternehmenskultur lange dauert, und man sich für einen gewissen Zeitraum auch mit „weichen Faktoren“ zufrieden geben muss.

\section{$5 \quad$ Transfer in andere Bereiche des Unternehmens}

Nach den ermutigenden ersten Sitzungen des Arbeitskreises gab es rasch eine Diskussion im Unternehmen über die Ausweitung der Gruppenarbeit. Angesichts der härter werdenden Marktbedingungen sollte nicht gewartet werden, bis umfassende Bewertungen der neuen Form der Arbeitsorganisation vorlagen. Zudem war man vom 
Prinzip der Veränderung überzeugt. Es gab gar Stimmen, die auf raschen gleichzeitigen Transfer in alle produzierenden Bereiche drängten. Schließlich wurde einem Konzept der geplanten und zügigen, aber schrittweisen Übertragung in die anderen Werksbereiche der Vorzug gegeben.

Dabei sind die generelle Vorgehensweise definiert, in jedem neuen Arbeitssystem müssen aber viele Fragen der konkreten Ausgestaltung jeweils neu und individuell entwickelt werden. Nur bedingt kann auf standardisierte Unterlagen zurückgegriffen werden. Wo möglich, wurden diese entwickelt. Damit hängt der Erfolg der flächendeckenden Verbreitung von Gruppenarbeit stark von der Qualifikation der jeweiligen Teamleiter und der dortigen Prozessbegleiter ab. Die dafür notwendigen Qualifikationen sind allerdings vorab erlernbar. Es wurde daher ein zweistufiges Verfahren entwickelt:

- Ein eigens konzipiertes Seminar für Prozessbegleiter vermittelt das notwendige Wissen über Gruppenarbeit, Vorgehen und Aufgaben des Prozessbegleiters.

- Die Verbreitung selbst findet in einer Art Schneeballverfahren statt. Die erste Übertragung fand im Team „Entfetten-Glühen“ selbst statt, an den Haubenglühen. Dort ist die Mannschaft eine andere, die Prozessbegleiter, die Schichtkoordinatoren und natürlich die Teamleitung jedoch dieselben Handelnden. An den Sitzungen des dort eingerichteten Arbeitskreises nehmen nun auch die Prozessbegleiter teil, die in ihrem Team als nächstes Gruppenarbeit einführen sollen. Dadurch erleben sie den Entwicklungsprozess nicht nur in der Theorie, sondern auch ganz praktisch.

So vorbereitet findet dann der nächste Übertragungsschritt statt. Nach und nach wird Arbeitssystem für Arbeitssystem in die Gruppenarbeit einbezogen. Der gesamte Einführungsprozess dauert so zwar länger als bei gleichzeitiger paralleler Einführung, ist aber der sicherere Weg und verhindert auch eine Überlastung der betrieblichen Organisation insgesamt. An der Überlegenheit der neuen Organisationsform gegenüber den alten Organisationsstrukturen gibt es im Unternehmen keinen Zweifel. Damit sie sich auf Dauer etabliert und mit Leben erfüllt bleibt, muss sie von den Beschäftigten angenommen werden und einen spürbaren Beitrag am ökonomischen Erfolg haben.
Leo Becker

Personalleiter Team 3

Rasselstein Hoesch $\mathrm{GmbH}$,

Koblenzer Str. 141

D-56 626 Andernach

Dr. Uwe Jürgenhake

Geschäftsführer

Soziale Innovation research \& consult $\mathrm{GmbH}$

Evinger Platz 17

D-44339 Dortmund

Christian Paqué

Prozeßbegleiter

Rasselstein Hoesch GmbH

Koblenzerstr. 141

D-56626 Andernach

Peter Schnittfeld

Wiss. Mitarbeiter

sfs Landesinstitut Sozialforschungsstelle

Evinger Platz 17

D-44339Dortmund 